\title{
Prolonged Abstinence and Changes in Alcoholic Personality: A NEO PI-R Study
}

\author{
Isabelle Boulze ${ }^{*}$, Michel Launay ${ }^{1}$, Bertrand Nalpas ${ }^{2}$ \\ ${ }^{1}$ Laboratory EA4556 Epsylon, Dynamics of Human Abilities \& Health Behaviors, Department of Medicine, \\ Subject and Society Sciences, Sport Sciences, University of Montpellier and St-Etienne, Montpellier, France \\ ${ }^{2}$ Inserm Département d'Information Scientifique et de Communication (DISC), Service de Médiation \\ Scientifique, Mission Inserm Associations, Paris, France \\ Email: ${ }^{*}$ isabelle.boulze@univ-montp3.fr
}

Received 22 January 2014; revised 23 February 2014; accepted 19 March 2014

Copyright (C) 2014 by authors and Scientific Research Publishing Inc.

This work is licensed under the Creative Commons Attribution International License (CC BY). http://creativecommons.org/licenses/by/4.0/

(c) () Open Access

\section{Abstract}

Many studies have examined the risk factors for relapse in alcohol-dependent patients within the first year of treatment, and have generally focused on two personality dimensions: emotional instability and difficulty in establishing relationships. In this study, we examine if these weaknesses remain in prolonged alcohol abstinence. To do so, we administer the NEO PI-R to two groups of subjects. Group 1, Inactive Drinkers (ID), consists of 51 patients with at least two years of abstinence (average length of abstinence for this group is 6.2 years); Group 2, Recently Detoxified Drinkers (RDD), comprises 93 patients who have recently ceased consuming alcohol. Based on NEO PI-R scores, our results evidence that inactive drinkers experience significant reduction in emotional instability and improvement in relationships to others. We further observe that, with long-term abstinence, these personality dimensions normalize, ceasing to be risk factors for relapse. Additionally, we find that this long-term amelioration of traits altered by alcohol amounts to an improved behavioral adaptation to life events rather than an actual change in personality.

\section{Keywords}

Alcoholism; Neo Pi-R; Personality; Abstinence; Emotional Stability; Relationship to Others

\section{Introduction}

Alcohol dependence is a chronic disease characterized by a high rate of relapse following withdrawal; indeed,

${ }^{*}$ Corresponding author. 
one year after detox, prolonged abstinence is achieved by only $20 \%$ to $30 \%$ of patients, while others return to heavy drinking, either occasionally or regularly (Hayashida et al., 1989; Gual et al., 1999). This high relapse rate is generally related to several medical or social causes, and traceable mainly to psychological defects. Restoring healthy emotions management is therefore one of the keys to prolonging abstinence. To be effectively established, self-defense strategies against challenging situations require adaptive modifications of personality traits. Few studies on personality changes related to alcohol withdrawal have been conducted (Mischel, 1968; Mac Adams, 1992; Bottlender \& Soyka, 2005); none of them have evidenced any significant, stable change, except for an improvement in emotional stability (Coëffec, 2011). Personality is defined as "the set of stable emotional and dynamic characteristics conditioning the personal modalities of reaction against a specific situation" (Bloch, 1999). A convenient instrument for analyzing personality is the Revised NEO Personality Inventory (NEO PI-R; Costa \& McCrae, 1992; NEO PI-R questionnaire Coëffec, 2011). This widely-used tool was validated with different populations (Pleasant et al., 2009) and the results showed good concordance agreement (Coëffec, 2011).

Patients with alcohol problems who were administered the NEO PI-R generally obtained a high "neuroticism" score (emotions, stress), associated with a low "agreeableness" score (relationship to others; Loukas et al., 2000). In the same vein, low "conscientiousness" scores (determination) were reported in patients who had abstained from alcohol for short periods (6 months to 1 year; Coëffec, Romo, \& Strika, 2009; Martin \& Sher, 1994; McCormick et al., 1998; Repetti et al., 2002). These data originally resulted from cross-sectional studies on alcoholic patients, and were later confirmed in a longitudinal study with a 6-month to 12-month follow-up (Bottlender \& Soyka, 2004). Conclusions converged, and the authors identified a common psychological profile for alcoholic subjects regardless of personal circumstances; moreover, these common psychological traits appeared to be stable through time, at least up to 12 months after alcohol consumption cessation.

Long-term, definitive abstinence remains a realistic objective for alcoholic patients, even if only a small proportion of them may achieve it. We therefore asked whether personality changes might occur long after alcohol withdrawal. To answer this question, we analyzed NEO PI-R personality traits in patients having achieved long-term abstinence, and compared them to those of newly detoxified patients.

\section{Methods}

\subsection{Subjects}

Two groups of subjects were assembled from a pool of volunteer patients. Inclusion criteria were: age 18 years and over, ability to speak and understand French; exclusion criteria were: active drug consumption, opiate substitution treatment, serious psychiatric comorbidity (psychosis, bipolarity, severe depression, generalized anxiety disorder) or life-threatening organic pathology.

The first group consisted of previously alcohol-dependent subjects who had been rigorously abstinent for at least two years. They were recruited from alcohol treatment centers or self-help groups, and were referred to as "Inactive Drinkers" (ID). The second group consisted of individuals who consulted for alcohol detoxification either for the first time, or following a relapse after a break of at least 6 months; this group was named "Recent Detox Drinkers" (RDD). Patients were recruited from seven clinics specialized in the treatment of addictions, located in the French Languedoc-Roussillon cities of Bagnols-sur-Cèze, Beziers, Grau-du-Roi, Montpellier, Narbonne, Nîmes and Vigan. To prevent gender bias, inclusion in the ID group was predicated on a sex ratio of two males to one female, as is usually prevalent in treatment centers for addiction to alcohol.

\subsection{Materials and Procedure}

All selected subjects were interviewed face-to-face by our researcher. Socio-demographic, medical and alcohol addiction data were collected through interviews and supplemented when necessary with medical records. Data included sex, marital status, socio-professional category, employment status, education level, quantity and length of alcohol consumption, number of prior treatments, history of hospitalization in psychiatry units and severity of associated pathologies. Alcohol dependence was assessed with the ICD-10 (1992).

Psychological data were collected using the self-administered NEO PI-R personality questionnaire (Costa \& McCrae, 1998, 2004). Based on advanced factor analysis (Cattell, 1996; Costa \& McCrae, 1998; Hogan, 2007; Hough \& Ones, 2003), this questionnaire includes 240 questions, exploring five personality domains with six 
facets each (Table 1).

The five domains are Neuroticism or Emotional Stability, Extraversion, Openness to Experience, Agreeableness (or Usability) and Conscientiousness (or Reliability). The analysis can be refined using data obtained with the 30 facets of these five key domains.

The NEO PI-R provides a self-scoring answer sheet: summing the raw scores for the item facets and for the main domains yields an overall score. Raw scores are then converted into standard scores. A graphic representation of the overall results can also be made to obtain an individual profile. According to Costa and McCrae (2005), personality traits are distributed in a Gaussian mode across 5 levels: very low $(\mathrm{T} \leq 34)$, low $(34 \leq \mathrm{T} \leq$ $44)$, medium $(45 \leq \mathrm{T} \leq 55)$, high $(56 \leq \mathrm{T} \leq 65)$, very high ( $\mathrm{T}>65)$; the scores must then be interpreted as indicators of personality traits without pathological significance.

\subsection{Statistical Analysis}

To ensure the anonymity and confidentiality of the data, subjects were identified solely by an inclusion number and their location source.

Quantitative data were analyzed using mean, standard deviation and median, and compared with the Student's t-test, or Wilcoxon $\mathrm{T}$ test when necessary; qualitative data were assessed with frequency and percentiles, and compared with the Chi Square Test or Fisher's Exact Test. Statistical analysis was performed using STATISTICA ( ) Version 7.1 software.

\section{Results}

\subsection{Socio-Demographics}

The ID group numbered 51 subjects; the RDD group totaled 93. Their main characteristics are shown in Table 2. Mean age was significantly higher in the ID group than in the RDD group, $F(1,139)=27.8, p<.001$. However, there was no age difference according to gender in either group, $F(1,139)=<1, p>.05$. The proportion of retirees was higher (37.2\% vs. $11.8 \%$ ) and that of the unemployed was lower (7.8\% vs. $19.3 \%)$ in the ID group than in the RDD group. No difference between groups was observed regarding sex ratio, marital status, socio-economic status or education level (Table 2).

\subsection{Alcohol Addiction}

The average length of abstinence in the ID group was 6.2, SD: 4.1 years (from two years to 17 years of abstinence; see Table 3). The average number of years of heavy drinking was significantly higher in the ID group than in the RDD group, $F(1,116)=8.49, p=.004$, and so was the number of previous treatments. In contrast, ID subjects reported significantly fewer alcohol-related diseases than RDD subjects $(17.6 \%$ vs. $32.2 \%$, $X 2$ with $4, d f=15.76, p=0.03)$. Nearly two thirds of patients in both groups reported a family history of alcoholism. Just over a quarter (26\%) of ID subjects reported having registered in psychiatric hospitals in the past, against $14 \%$ of RDD subjects; however, the difference was not statistically significant.

\subsection{Psychological Data}

NEO PI-R standard note (T) results for both ID and RDD groups are presented in Table 4; the corresponding

Table 1. NEO PI-R domains and associated facets (McCrae et al., 1998).

\begin{tabular}{lr}
\multicolumn{1}{c}{ Domains } & \multicolumn{1}{c}{ Facets } \\
\hline $\begin{array}{l}\text { 1. Neuroticism } \\
\text { 2. Extraversion }\end{array}$ & Anxiety, Hostility, Depression, Self-Consciousness, Impulsiveness, Vulnerability to Stress \\
$\begin{array}{l}\text { 3. Openness to Experience } \\
\text { 4. Agreeableness }\end{array}$ & Fantasy, Aesthetics, Feelings, Actions, Ideas, Values \\
\begin{tabular}{l} 
5. Conscientiousness \\
\hline
\end{tabular} & Competence, Order, Dutifulness, Achievement Striving, Self-Discipline, Deliberation \\
\hline
\end{tabular}


Table 2. Socio-demographic characteristics of Inactive Drinkers (ID) and Recently Detoxified Drinkers (RDD).

\begin{tabular}{|c|c|c|c|}
\hline & ID & RDD & $p$ \\
\hline $\mathbf{N}$ & 51 & 93 & \\
\hline Sex Ratio (M/F) & 2 & 0.73 & \\
\hline Age (M+/-SD) & 54.7 & 45.8 & $<0.001$ \\
\hline \multicolumn{4}{|l|}{ Lifestyle } \\
\hline Singles (\%) & 54.9 & 34.4 & NS \\
\hline Couples (\%) & 45.1 & 65.6 & \\
\hline \multicolumn{4}{|l|}{ Socioeconomic Status } \\
\hline Lower Class (\%) & 73.8 & 70.3 & \\
\hline Middle Class (\%) & 18.4 & 14.8 & NS \\
\hline Upper Class (\%) & 7.8 & 14.8 & \\
\hline \multicolumn{4}{|l|}{ Professional Status } \\
\hline Employed (\%) & 35.3 & 33 & \\
\hline Unemployed (\%) & 7.8 & 19.3 & NS \\
\hline Retired (\%) & 37.2 & 11.8 & \\
\hline Disabled (\%) & 11.7 & 6.4 & \\
\hline \multicolumn{4}{|l|}{ Education } \\
\hline Primary (\%) & 53 & 47.8 & \\
\hline Secondary (\%) & 26.5 & 28.2 & NS \\
\hline Graduate and Advanced (\%) & 18.3 & 23.9 & \\
\hline
\end{tabular}

Table 3. Length of abstinence, alcohol consumption, and family history of alcoholism of Inactive Drinkers (ID) and Recently Detoxified Drinkers (RDD).

\begin{tabular}{ccc}
\hline & ID & RDD \\
Abstinence (Mean \pm SD, Years) & $6.2+/-4.1$ & $<=0.1$ \\
Duration of Alcohol Consumption (Mean \pm SD, Years) & $19.7+/-8.5$ & $14.2+/-9.4$ \\
Family History of Alcoholism (\%) & 65.9 & 0.004 \\
\hline
\end{tabular}

Table 4. T score means and standard deviations of Inactive Drinkers (ID) and Recently Detoxified Drinkers (RDD) for each NEO PI-R domain.

\begin{tabular}{|c|c|c|c|c|c|}
\hline \multirow[b]{2}{*}{ Group } & \multicolumn{5}{|c|}{ NEO PI-R Domains (M \pm SD) } \\
\hline & Neuroticism & Extraversion & Openness & Agreeableness & Conscientiousness \\
\hline ID & $51.8 \pm 10.1$ & $48.6 \pm 8.5$ & $47.1 \pm 8.8$ & $53.2 \pm 9.2$ & $52.2 \pm 8.5$ \\
\hline RDD & $58.2 \pm 8.7$ & $46.9 \pm 8.9$ & $47 \pm 10.6$ & $49.7 \pm 9$ & $46.6 \pm 10.3$ \\
\hline$p$ & .0001 & .28 & .98 & .029 & .001 \\
\hline
\end{tabular}

personality profiles are shown in Figure 1. Inactive drinkers displayed a "medium” NEO PI-R profile: their neuroticism, agreeableness and conscientiousness scores were in the upper part of the medium range; their 


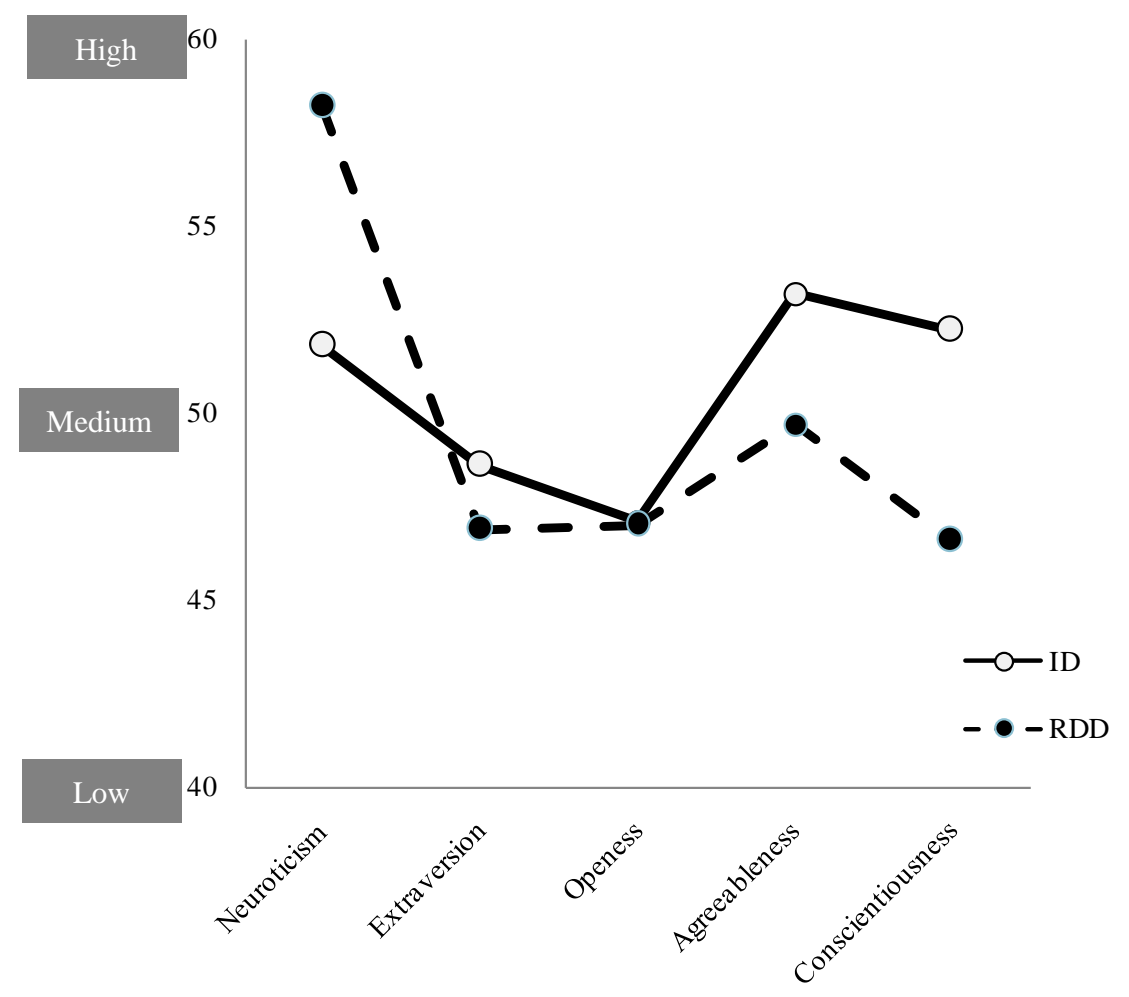

Figure 1. NEO PI-R profiles of Inactive Drinkers (ID) and Recently Detoxified Drinkers (RDD).

extraversion and openness scores were in the lower part. Recently detoxified drinkers obtained scores similar to those of the ID group subjects for extraversion (m: 46.9, SD: 8.9 vs. m: 48.6, SD: 8.5, NS) and openness (m: 47.0, SD:10.6 vs. m: 47.1, SD: 8.8, NS); however, they scored in the high range of the usual neuroticism value and their score (m: 58.2, SD: 8.7) was significantly $(p=0.0001)$ higher than that of inactive drinkers $(\mathrm{m}: 51.8$, SD: 10.1); conversely, although in the medium range, RDD scores for agreeableness and conscientiousness were significantly lower than ID scores (m: 49.7, SD: 9 vs. m: 53.2, SD: 9.2, $p=0.03$ and m: 46.6, SD: 10.3 vs. m: 52.2, SD: $8.5, p=0.001$, respectively).

The three domains in which ID and RDD exhibited significant differences (i.e., neuroticism, agreeableness and conscientiousness) were further analyzed according to their facets. Neuroticism was assessed with anxiety, anger-hostility, depression, self-consciousness, impulsiveness, and vulnerability to stress; ID and RDD subjects differed significantly on five of those traits, but scored similarly on self-consciousness. For conscientiousness, ID and RDD subjects differed significantly on 4 facets: competence, sense of duty, self-discipline and deliberation; however, they did not differ on achievement striving and order. Finally, for agreeableness, ID and RDD subjects differed significantly on modesty, but scored similarly on trust, straightforwardness, altruism, compliance and tender mindedness.

\section{Discussion}

This study aimed to examine whether personality traits are modified during prolonged abstinence in formerly alcohol-dependent patients. To do so, we administered the NEO PI-R questionnaire to long-term abstinent subjects (inactive drinkers) and recently detoxified patients (recently detoxified drinkers). NEO PI-R scores indicated that inactive drinkers differed significantly from recently detoxified ones in three personality domains: neuroticism $(p=.001)$, agreeableness $(p=.029)$ and conscientiousness $(p=.001)$. In other words, these domains are discriminative.

Regarding neuroticism, we found that inactive drinkers do not necessarily focus on negative issues. They are not shy in the presence of others and remain in control of their emotions, thusly handling frustrations better (thereby enhancing their ability to remain abstinent). Inactive drinkers are able to cope with stress and manage 
challenging situations without letting their emotions overrun them. Conversely, recently detoxified drinkers scored high on neuroticism (58.2). They experience difficulty in adjusting to events, a dimension which is associated with emotional instability (stress, uncontrolled impulses, irrational ideas, negative affect). Socially, they tend to isolate themselves and to withdraw from social relationships, preferring instead a hedonistic lifestyle, as suggested by several authors (McCrae et al., 1986). These results matched those found the literature (Loukas et al., 2000).

Regarding agreeableness (which ties back into social relationships), we found that inactive drinkers care for, and are interested in, others (altruism); they consider that helping others may lead to receiving help in return. Instead, recently detoxified drinkers' low self-esteem and narcissism prevent them from enjoying interpersonal exchanges, and lead them to withdraw from social relationships.

Finally, regarding conscientiousness, we observed that, over time, inactive drinkers become more social, enjoy higher self-esteem (Costa, McCrae, \& Dye, 1991), care for and are interested in others, and wish to help them (with the assumption that they will receive help in return). They are able to perform tasks without being distracted, and carefully consider their actions before carrying them out; their determination remains strong regardless of the level of challenge, and their actions are guided by ethical values. Instead, recently detoxified drinkers lack confidence, rush into action, prove unreliable and unstable. As a result, lacking sufficient motivation, they experience difficulty in achieving their objectives.

Hence, in the above psychology domains, our results evidenced significant differences between the two groups. Inactive drinkers seem less nervous, less angry, less depressed, less impulsive and less vulnerable than recently detoxified drinkers. Inactive drinkers' level of competence, sense of duty, self-discipline and ability to think before acting increases with time. While previous studies have generally focused on shorter lengths of abstinence (Martin \& Sher, 1994; McCormick et al., 1998; Repetti et al., 2002; Bottlender \& Soyka, 2004), ours differed in that it examined long-term abstinent subjects (2 years or more) and revealed a marked improvement in specific domains over time. Indeed, these results are quite encouraging for alcoholic patients, who may aspire to greater quality of life through long-term abstinence.

However, in spite of marked differences between groups, our results did not provide clear evidence of personality changes. While significant behavior differences between the two groups were revealed, they were more akin to long-term improvements in behavorial adequacy to events than to actual personality changes. Indeed, upon examination of the scores' distribution across the 5 personality domains, we observed that it lied in the same medium range $(45 \leq \mathrm{T} \leq 55)$ for 4 of them; neuroticism scores were the only ones to "normalize", i.e., to move from a high level for recently detoxified drinkers to a medium level for inactive drinkers. These observations underscore the non-pathological nature of the psychological issues affecting alcoholic patients, and the latter's potential for stabilization over time by overcoming previous behavior weaknesses. Hence, this process is not one of personality change, but rather one of better adequacy of behavior responses to reality and its changing parameters.

Several significant elements were highlighted in our study. First, it evidenced the psychological differences between inactive drinkers and recently detoxified drinkers. Second, it noted the absence of predictors of short-term or long-term success of abstinence at the time of initial treatment request. Indeed, neither did socio-demographic factors, nor the bulk of the anamnesis data, nor even personality factors seem to allow prognosis. Third, it revealed that treatment-induced behavior changes showed a decrease in neuroticism and an increase in traits related to responsibility and conscientiousness. These trends could eventually provide the basis for predictors of success of abstinence treatments.

When interpreting our results, one must remain aware that our study was not longitudinal, but based instead on the cross-comparison of two independent groups. Nonetheless, the results suggest that alcoholic patients (excluding those suffering from major psychiatric pathologies-see our exclusion criteria) possess personal traits similar to those of the general population, except for heightened neuroticism shortly after alcohol consumption cessation. In fact, these results undermine the persistent notion that alcoholic patients exhibit specific vulnerability factors. Abstinence seems associated with a behavioral improvement to adequately respond to life events, but without making actual personality modifications. These observations underscore the non-pathological nature of the psychiatric issues facing alcoholic patients and the latter's potential for stabilization over time by overcoming previous behavioral weaknesses.

Our results, obtained from a sample of over one hundred subjects, therefore suggest that the personality of alcoholic patients remains similar to that of individuals from the general population. Active drinkers exhibit more 
neuroticism, less agreeableness and less conscientiousness than long-term teetotalers. Long-term teetotalism seems to foster a clear amelioration of the personality traits impaired by alcohol, but not a modification of personality. The rational management of emotions appears to be the single key factor of lasting abstinence, via the restoration or the new establishment of defense mechanisms required for coping with challenging situations, and thus seems likely to impose behavior modifications. By focusing on emotions management and its effects on behavior, further studies could eventually identify relevant indicators of treatment success.

\section{Acknowledgements}

This study was funded by a grant from the MILDT (Mission Interministérielle de Lutte contre la Drogue et al Toxicomanie-French Interministerial Office for the Fight against Drugs and Toxicomania).

\section{References}

Bloch, H., Chemama, R., \& Dépret, E. (1999). Large Dictionary of Psychology. Paris: Larousse, Paris.

Bottlender, M., \& Soyka, M. (2005). Impact of Different Personality Dimensions (NEO Five-Factor Inventory) on the Outcome of Alcohol-Dependent Patients 6 and 12 Months Effective Treatment. Psychiatry Research, 15, 61-67.

http://dx.doi.org/10.1016/j.psychres.2004.07.013

Cattell, H. E. P. (1996). The Original Big Five: A Historical Perspective. European Review of Applied Psychology, 46, 5-14.

Coëffec, A. (2011). Model Inputs of the Five Major Factors in the Field of Alcohol. The Brain, 37, 75-82.

Coëffec, A., Romo, L., \& Strika, L. (2009). Personalité et impulsivité: étude portant sur 20 patients alcoolodépendants. Alcoologie et Addictologie, Présentation, 31, 327-332.

Costa Jr., P. T., \& McCrae, R. R. (1998). NEO PI-R. Manual. Paris: Editions du Centre of Applied Psychology.

Costa Jr., P. T., \& McCrae, R. R. (2005). NEO PI-R Personality Inventory-Revised. Manual Paris: Editions du Centre of Applied Psychology.

Gual, A., Lligona, A., \& Colom, J. (1999). Five-Year Outcome in Alcohol Dependence. A Naturalistic Study of 850 Patients in Catalonia. Alcohol Alcoholism, 34, 183-192. http://dx.doi.org/10.1093/alcalc/34.2.183

Hayashida, M., Alterman, A. I., McLellan, T., Purtill, J. J., O’Brien, C. P., Volpicelli, J. R., Raphaelson, A. H., \& Hall, C. P. (1989). Comparative Effectiveness and Costs of Inpatient and Outpatient Detoxification of Patients with Mild-to-Moderate Alcohol Withdrawal Syndrome. New England Journal of Medicine, 320, 358-364. http://dx.doi.org/10.1056/NEJM198902093200605

Hogan, J., Barrett, P., \& Hogan, R. (2007). Personality Measurement, Faking, and Employment Selection. Journal of Applied Psychology, 92, 1270-1285. http://dx.doi.org/10.1037/0021-9010.92.5.1270

Hough, L. M., \& Ones, D. S. (2001). The Structure, Measurement, Validity, and Use of Personality Variables in Industrial, Work, and Organizational Psychology. In N. Anderson, D. S. Ones, H. K. Sinangil, \& C. Viswesvaran (Eds.), Handbook of Industrial, Work, and Organizational Psychology (pp. 233-277). London: Sage.

Loukas, A., Krull, J. L., \& Chassin, L. (2000). The Relationship of Personality to Alcohol Abuse/Dependence in a HighRisk Sample. Journal Personality, 68, 1153-1175. http://dx.doi.org/10.1111/1467-6494.00130

Martin, E. D., \& Sher, K. J. (1994). Family History of Alcoholism, Alcohol Use Disorders and the Five-Factor Model of Personality. Journal Study Alcohol, 55, 81-90.

McCormick, R. A., Dowd, E. T., \& Quirk, S. (1998). The Relationship of NEO PI Performance to Coping Styles, Patterns of Use and Triggers for Use among Substance Abusers. Addictive Behaviors, 18, 497-507.

http://dx.doi.org/10.1016/S0306-4603(98)00005-7

McCrae, R. R., \& Costa Jr., P. T. (2004). A Contemplated Revision of the NEO Five-Factor Inventory. Personality and Individual Differences, 36, 587-596. http://dx.doi.org/10.1016/S0191-8869(03)00118-1

McCrae, R. R., Costa Jr., P. T., \& Busch, C. M. (1986). Evaluating Comprehensiveness in Personnality Systems: The California Psychological Inventory and the Five-Factor Model. Journal of Personality, 61, 1-26. http://dx.doi.org/10.1111/j.1467-6494.1993.tb00276.x

McCrae, R. R., Costa Jr., P. T., Pilar, G. H., Rolland, J. P., \& Parker, W. D. (1998). Cross-Cultural Assessment of the FiveFactor Model: The Revised NEO Personality Inventory. Journal of Cross-Cultural Psychology, 29, 71-188. http://dx.doi.org/10.1177/0022022198291009

Mischel, W. (1968). Personality and Assessment. New York: Witley.

Repetti, R., Taylor, S., \& Seeman, T. (2002). Risky Family Social Environments and Physical and Mental Health of the Offspring. Psychology Bulletin, 128, 330-366. http://dx.doi.org/10.1037/0033-2909.128.2.330 
Sinangil, D., \& Viswesvaran, C. (2002). Handbook of Industrial, Work and Organizational. Psychology, Personnel Psychology, 1, 233-267. 